\title{
Eficiência técnica e heterogeneidade tecnológica na agropecuária das regióes semiárida e não semiárida do Nordeste brasileiro
}

\author{
Technical efficiency and technological heterogeneity in \\ agriculture in the semi-arid and non-semi-arid regions of \\ northeastern Brazil
}

\author{
Felipe Pinto da Silva ${ }^{1}$ (D), Jair Andrade Araujo ${ }^{2}$ (D), Edward Martins Costa ${ }^{2}$ \\ e José Eustáquio Ribeiro Vieira Filho ${ }^{3}$
}

\begin{abstract}
Resumo: Este estudo busca analisar as diferenças tecnológicas da produção agropecuária entre os municípios situados no semiárido e no não semiárido do Nordeste brasileiro. Utiliza-se o modelo de meta-fronteira tecnológica, proposta por Battese, Rao e O'Donnell (2004) (2004) e O'Donnell et al. (2008), para responder até que ponto essas diferenças regionais influenciam na eficiência da produção. Constatou-se que a hipótese de que os municípios pertencentes ao semiárido e ao não semiárido deparam-se com diferentes oportunidades de produção. Os resultados indicaram que, quando comparadas as duas regiões, o não semiárido possui a maior eficiência técnica média com referência à meta-fronteira.
\end{abstract}

Palavras-chave: agricultura, tecnologia, semiárido nordestino, fronteira de produção.

\begin{abstract}
This study aims to analyze the technological differences in agricultural production between the municipalities in the semiarid and non-semiarid Northeast Brazil. It uses the technological meta-frontier model proposed by Battese, Rao e O'Donnell (2004), and O'Donnell, Rao e Battese (2008), for responding to what extent these regional differences influence the efficiency of production. It was observed that the hypothesis that the municipalities belonging to the semiarid and non-semiarid face with different production opportunities. The results indicated, that comparing both regions, the non-semiarid region has the highest average technical efficiency with reference to meta-frontier.
\end{abstract}

Key-words: agriculture, technology, semi-arid northeast, production frontier.

Classificação JEL: D24, Q10.

Data de submissão: 21 de março de 2017. Data de aceite: 28 de julho de 2018.

1. Universidade Estadual de Campinas (UNICAMP), Campinas (SP), Brasil. E-mail: dasilva.felipe0@gmail.com

2. Universidade Federal do Ceará (UFC), Fortaleza (CE), Brasil. E-mails: jaraujoce@gmail.com; edwardcosta@ufc.br

3. Instituto de Pesquisa Econômica Aplicada (IPEA), Brasília (DF), Brasil. E-mail: jose.vieira@ipea.gov.br 


\section{Introdução}

O Brasil passou por um processo intenso de modernização do setor agrícola a partir da década de 1960. Gasques et al. (2012) apresentam evidências do crescimento elevado da produtividade agrícola nacional, com taxas superiores às dos principais países produtores de grãos e carnes, tais como Estados Unidos, União Europeia, China, Índia e Argentina. De forma complementar, Fuglie \& Wang (2016) constataram que a agricultura brasileira e a chinesa apresentaram, nos últimos 20 anos, o maior e mais rápido crescimento da produtividade total dos fatores (PTF) em âmbito mundial. Além disso, essa tendência se intensificou durante o período de 2000 a 2012 (Gasques et al., 2012).

Esse processo de modernização, contudo, instaurou-se de maneira heterogênea dentre as regiões brasileiras, dado que a pobreza rural e a agricultura de subsistência de baixa produtividade persistem, principalmente no semiárido nordestino (Barbieri et al., 2010). Diante dessas particularidades, o setor agrícola, situado nas diferentes regiões do Brasil, depara-se com diferentes bases institucionais e tecnológicas, condições geográficas, climáticas que podem aprofundar a heterogeneidade produtiva da região.

No Brasil, o Nordeste é uma das áreas mais vulneráveis às variabilidades climáticas, que se associam às chuvas irregulares, aos déficits hídricos e à baixa resiliência da produção agrícola das populações que se dedicam à agricultura, principalmente nos assentamentos da região semiárida (Simões et al., 2010). Estas características fazem da agropecuária um empreendimento cercado de riscos, especialmente no semiárido. Ao contrário da dinâmica da moderna produção, o setor agropecuário constituído no semiárido nordestino tem acesso restrito aos mercados, o que dificulta o desenvolvimento regional.

Vale observar que, mesmo no Nordeste, o setor agrícola não se comporta de maneira homogênea. Agricultores situados no semiárido, por hipótese, deparam-se com condições geográficas, culturais, históricas e institucionais distintas, que devem ser consideradas nas análises de produtividade e eficiência técnica. Estudos com essa natureza são de grande valia para avaliação de políticas públicas de desenvolvimento regional. É necessário promover as instituições de forma que não haja o aprofundamento da discrepância produtiva entre a região dinâmica e atrasada, como bem demonstram os estudos de Hayami (1969) e Hayami \& Ruttan (1970; 1971).

Assim, pode-se levantar alguns problemas: $i$ ) essas diferenças influenciam na eficiência da produção?; e ii) se positiva a resposta ao primeiro questionamento, qual a magnitude dos impactos produtivos em pertencer ou não à uma dada região? Espera-se que esta problemática seja respondida ao longo do estudo. Dadas estas questões, a hipótese deste estudo é que o setor agropecuário do Nordeste se comporta de maneira heterogênea, em parte relacionada pela extensa área semiárida na região. Expostas as questões centrais, como objetivo geral de investigação, busca-se identificar as diferenças tecnológicas da produção agropecuária entre os municípios situados no semiárido e nas demais regiões do Nordeste brasileiro. Para tanto, propõe-se a 
utilização do método utilizado por Battese et al. (2004) e O’Donnell et al. (2008) para estimação da meta-fronteira de produção. Este quadro teórico é bastante utilizado para a comparação de níveis de eficiência e tecnologias de produção entre regiões distintas.

Serão observadas em que proporção as diferenças regionais próprias do Nordeste, no que tange à qualidade do solo, diferença climática, infraestrutura econômica, dentre outras variáveis, impactam na eficiência das firmas situadas nos diferentes grupos regionais. Particularmente, a análise se concentra no estudo do Nordeste, incluindo o semiárido e as demais regiões, a partir da cartografia delimitada pela Superintendência do Desenvolvimento do Nordeste (Sudene). Como objetivo específico, procura-se mensurar e analisar a eficiência técnica (ET) e a razão de meta-fronteira (MRT) dos municípios inseridos no semiárido e não semiárido nordestino.

A principal contribuição deste estudo consiste em melhor caracterização dos municípios situados no semiárido e nas demais regiões do Nordeste brasileiro, atentando-se para as questões produtiva e tecnológica. A literatura nacional que trabalha com eficiência produtiva na agropecuária por meio de fronteira estocástica de produção apresenta uma lacuna em relação à diferenciação tecnológica existente entre as regiões brasileiras e seus impactos na eficiência produtiva da agropecuária. Segundo Orea \& Kumbhakar (2004), estimativas de eficiência técnicas em modelos de fronteira estocástica normalmente assumem que a tecnologia de produção subjacente é a mesma para todas as empresas. Assim, diferenças tecnologicas não observadas podem ser inapropriadamente identificadas como ineficiência, caso a heterogeneidade tecnologica existente entre as firmas (municípios) não for levada em consideração.

Para a análise, serão apresentadas cinco seções, incluindo esta breve introdução. Na seção 2 situa-se a fundamentação teórica para o presente estudo, fazendo-se uma breve caracterização do semiárido, além de compilar estudos empíricos relacionados à eficiência produtiva da agropecuária da região. A seção 3 apresenta a metodologia e o modelo econométrico, bem como a fonte dos dados e a amostra dos municípios usados na estimação. Na seção 4, há a discussão dos resultados. Por fim, seguem as considerações finais.

\section{Fundamentação teórica}

\subsection{O Semiárido brasileiro como delimitação empírica}

O Nordeste brasileiro tem área total de 1,57 milhão de $\mathrm{km}^{2}$, perfazendo $18 \%$ do território nacional. Trata-se da segunda região mais populosa do País, constituindo $28 \%$ da população total do Brasil e concentrando cerca de $40 \%$ da pobreza. Em 2010, essa disparidade foi ilustrada ao comparar o índice de desenvolvimento humano $(\mathrm{IDH})^{1}$ de 0,57 da região Nordeste com o indicador da região Sudeste, de 0,78, como apontam Barbieri et al. (2010). Parcela significativa da população consiste de agricultores familiares tradicionais de subsistência e com baixo teor tecnológico.

Como já destacado, a região Nordeste é heterogênea, tanto economicamente quanto do ponto de vista da distribuição dos recursos naturais. A região semiárida, ocupando $60 \%$ de toda região Nordeste, estende-se a uma área de $982.563,3 \mathrm{~km}^{2}$, sendo que $89,5 \%$ pertencem ao Nordeste brasileiro e o restante, ao norte do estado de Minas Gerais. Em termos populacionais, o semiárido nordestino compreende aproximadamente $12 \%$ da população do País. Popularmente conhecida como o "polígono das secas", é a região mais pobre do Brasil, sendo sujeita a severas secas periódicas (Mata \& Resende, 2015), tornando-a a região semiárida dos trópicos mais populosa do mundo. (Marengo, 2008).

Em 2010, a região semiárida apresentava um nível de analfabetismo três vezes maior que a média nacional (Rocha \& Soares, 2015). Conforme mostrado na Tabela 1, ao comparar os indicadores demográficos, para 2010, a média da renda domiciliar per capita do semiárido nordestino foi 2,4 vezes menor em relação à média nacional, e apenas $70 \%$ da renda média do Nordeste.

Ao longo do período de 2000 a 2010, percebe-se que o semiárido foi a região analisada com maior variação percentual de renda domiciliar per capita, o que resultou,

1 Índice sintético calculado pela média simples de três subíndices, referentes às dimensões de longevidade (IDHM-L), educação (IDHM-E) e renda (IDHM-R). Para obter informações metodológicas, consulte o Atlas do Desenvolvimento Humano no Brasil, elaborado pelo Programa das Nações Unidas para o Desenvolvimento (PNUD, 1998). O IDH é uma medida comparativa para classificar diferentes regiões pelo grau de desenvolvimento. Quanto mais próximo de 1, mais elevado é o grau de desenvolvimento. Ao contrário, quanto menor for o indicador, mais frágil é o desenvolvimento humano da região. 
Tabela 1. Média da renda domiciliar per capita real e índice de Gini para o Brasil, Nordeste e semiárido nordestino (2000 e 2010)

\begin{tabular}{|c|c|c|c|c|c|c|}
\hline \multirow{2}{*}{ Unidades } & \multicolumn{3}{|c|}{ Renda (em R\$) } & \multicolumn{3}{|c|}{ Índice de Gini } \\
\hline & 2000 & 2010 & Variação (\%) & 2000 & 2010 & Variação (\%) \\
\hline Semiárido nordestino & 246,86 & 399,48 & 61,82 & 0,58 & 0,55 & $-5,17$ \\
\hline Nordeste & 365,89 & 570,10 & 55,81 & 0,64 & 0,62 & $-3,13$ \\
\hline Brasil & 701,71 & 949,99 & 35,38 & 0,63 & 0,60 & $-4,29$ \\
\hline
\end{tabular}

Fonte: Elaboração dos autores, a partir de Irffi (2015).

consequentemente, em melhora na distribuição de renda comparada às demais regiões. Deve-se ter cuidado ao interpretar o índice de $\mathrm{Gini}^{2}$ pois, embora a renda tenha aumentado na região Semiárida ao longo do período, os valores absolutos se mostram mais baixos que nas demais regiões. Essa maior igualdade de renda se dá em uma situação de homogeneização da pobreza na região Semiárida, como aponta Vieira Filho (2013), ainda que tenha existido melhora de renda. Esse resultado para a região Semiárida indica que há uma homogeneização das rendas da população residente na região em patamares baixos, como foi apresentado na seção anterior.

A distribuição populacional da região não acompanhou o processo de urbanização que ocorreu no Brasil no século passado (Alves \& Rocha, 2010). De acordo com os autores, o Nordeste é a região com maior potencial de êxodo rural nos próximos anos e, por isso, é necessário definir políticas que possam fomentar o espaço rural e o urbano. Os municípios que compõem o semiárido são pequenos, com população média de 12 mil habitantes, tendo sua economia fortemente baseada em formas extensivas de agricultura de subsistência com baixa produtividade e pouca resiliência às flutuações climáticas. (Barbieri et al., 2010).

A partir dos dados apresentados pelo Censo Agropecuário de 2006, do Instituto Brasileiro de Geografia e Estatística (Instituto Brasileiro de Geografia e Estatística, 2006), a agricultura familiar é o segmento predominante no contexto produtivo da agropecuária da região. De acordo

2 O coeficiente de Gini mede o grau de desigualdade na distribuição da renda domiciliar per capita entre os indivíduos. Seu valor pode variar teoricamente desde 0 , quando não há desigualdade (as rendas de todos os indivíduos têm o mesmo valor), até 1 , quando a desigualdade é máxima (apenas um indivíduo detém toda a renda da sociedade e a renda de todos os outros indivíduos é nula). Série calculada a partir das respostas à Pesquisa Nacional por Amostra de Domicílios do Instituto Brasileiro de Geografia e Estatística (Pnad/IBGE). com o Censo Agropecuário de 2006 (Instituto Brasileiro de Geografia e Estatística, 2006), dos 5,1 milhões de estabelecimentos agropecuários no Brasil, cerca de 84\% eram de agricultura familiar. No comparativo regional, dos estabelecimentos de agricultura familiar, quase a metade $(50 \%)$ estava localizada no Nordeste. No que se refere à extrema pobreza, essa porcentagem ainda é muito mais elevada na região nordestina: cerca de $61 \%$.

Diante disso, os formuladores de políticas públicas voltaram sua atenção a essas questões, criando políticas específicas para o enfrentamento dessa condição. O principal instrumento de intervenção governamental, representado pela Superintendência para o Desenvolvimento do Nordeste (Sudene), é o Fundo Constitucional do Nordeste (FNE), que tem como seu gestor o Banco do Nordeste Brasileiro (BNB). A Constituição Federal de 1988 estabeleceu o FNE e outros fundos constitucionais ${ }^{3}$ através do artigo 159 e regido por força da Lei n. 7.827, de 27 de setembro de 1989, sendo metade dos recursos dos fundos direcionados à região Semiárida.

A delimitação do Semiárido brasileiro é regulamentada pela Portaria n. 89, assinada em março de 2005 pelo Ministério da Integração Nacional. O Semiárido brasileiro constitui toda a área dos estados do Nordeste, incluindo todos os municípios do norte de Minas Gerais e do Espírito Santo que atendam aos seguintes critérios: precipitação pluviométrica média anual inferior a 800 milímetros; índice de aridez de até 0,5 calculado pelo balanço hídrico que relaciona as precipitações e a evapotranspiração potencial, no período de 1961 e 1991;

3 Os fundos constitucionais foram criados no âmbito da Constituição Federal de 1988 e são constituídos de 3\% da arrecadação do imposto sobre Renda e Proventos de Qualquer Natureza (IR) e do imposto sobre produtos industrializados (IPI). Os fundos são: Fundo Constitucional de Financiamento do Nordeste (FNE), Fundo Constitucional de Financiamento do Norte e Fundo Constitucional de Financiamento do Centro-Oeste (FCO). 
e risco de seca maior que $60 \%$, tomando-se por base o período de 1970 e 1990.

A importância desse fundo para a região foi mostrada por Mata \& Resende (2015). No estudo, os autores compararam os municípios incluídos na região Semiárida (grupo de tratamento), com municípios semelhantes que não foram incorporados à região na lei de 2005 (grupo de controle). Constatou-se que produtores de baixa renda e com menor porte nas novas localidades semiáridas têm investido o crédito subsidiado para expandir sua capacidade de resiliência às intemperes climáticas regionais.

Embora os municípios do norte de Minas Gerais pertençam a um estado rico, com maior grau de desenvolvimento, estes não se beneficiam desse nível de desenvolvimento, como mostra Irffi (2015). Já o norte do Espírito Santo foi mantido para garantir a estrutura institucional criada pela atuação da Sudene na região. Vale destacar que parte do Semiárido brasileiro está no Cerrado Nordestino ${ }^{4}$, área da nova expansão agrícola do Brasil, que vem se constituindo nos últimos 10, ou 15 anos. (Buainain \& Garcia, 2016).

Assim, dado o horizonte histórico deste estudo (1970-2006), optou-se por não se considerar este conjunto regional separadamente, já que esta região ainda não se enquadra no eixo mais dinâmico do setor nacional. Esse esforço busca captar o impacto da heterogeneidade espacial nordestina na eficiência das firmas de produção agropecuária situadas na delimitação proposta, e verificar o quanto as diferenciações tecnológicas entre as regiões influencia na eficiência agrícola dos municípios.

\subsection{Principais tendências verificadas}

Estudos que se detêm a analisar a evolução da PTF e da eficiência agrícola da região Semiárida são escassos. A maioria dos trabalhos considera apenas o Nordeste como um todo. Desconsiderando a elevada heterogeneidade regional, os agricultores fazem as suas escolhas a partir de diferentes "conjuntos de tecnologias". A literatura econômica sobre o tema mostra que o Nordeste vem à margem desse processo. Vicente (2004), ao analisar a eficiência e a PTF da agricultura brasileira no período de 1970 a 1995, utilizando Análise Envoltória de Dados (DEA),

4 O Cerrado Nordestino compreende parte do bioma Cerrado, que se localiza no Nordeste do Brasil, estendendo-se do Maranhão até o norte de Minas Gerais. indicou que o Nordeste teve uma involução tecnológica, o que o fez destoar do aumento da PTF pelo qual passou o setor agrícola brasileiro. Os principais geradores desta tendência foram a deficiência na infraestrutura local, a dificuldade no acesso e na utilização de insumos modernos e a deterioração das relações de troca na agricultura. A amálgama desses fatores influenciou o desempenho PTF na região.

Araújo \& Santos (2009) argumentam que o Nordeste passou por um processo tardio de integração à dinâmica da agricultura brasileira, passando a acompanhar de perto a economia brasileira como um todo, crescendo a taxas médias superiores às nacionais. A base agropecuária da região passou por um processo de diversificação. Toma-se como exemplo a presença da fruticultura irrigada, especialmente no polo agroindustrial de Petrolina-Juazeiro no vale submédio do São Francisco e no moderno polo de fruticultura do Rio Grande do Norte no Vale do Açu. Da mesma forma, a produção de grãos vem crescendo na direção do Matopiba, que se estende aos Cerrados nordestino e tocantinense.

Fornazier \& Vieira Filho (2012), ao estudarem o setor agropecuário brasileiro, encontraram fortes evidências de que o setor é estruturalmente heterogêneo. Para tanto, compararam as diferenças a partir dos dados do Censo Agropecuário de 2006. Isso significa que, no caso brasileiro, o setor moderno e produtivo convivia com o atrasado e pobre, bem representados na diferença entre os produtores familiares e não familiares. Entretanto, os autores encontraram que a heterogeneidade não se dá unicamente pelo tipo de produção (familiar ou patronal) ou mesmo pelo tamanho (pequeno ou grande). Há outras características como fatores culturais, geográficos e climáticos. Além do mais, foi constatado que, dentro da própria categoria de agricultura familiar, havia produção moderna com alta produtividade versus produção atrasada com baixa eficiência técnica produtiva, que produzem praticamente sem excedente monetário.

Vieira Filho (2013) verificou a heterogeneidade estrutural existente na agricultura familiar brasileira em diferentes regiões e constatou que há a coexistência de produtores familiares, modernos e produtivos, com agricultores tradicionais de subsistência com baixo teor tecnológico, sendo estes últimos mais vulneráveis às variabilidades climáticas próprias da região Nordeste. Apresentou que a região Nordeste tem a maior heterogeneidade entre os agricultores familiares, já que a maior parte de seus 
estabelecimentos agropecuários se classificava nesse tipo de organização: cerca de $88 \%$ do total (Instituto Brasileiro de Geografia e Estatística, 2006).

Helfand et al. (2015) analisaram a PTF e a eficiência técnica com o modelo de fronteira estocástica a partir dos dados dos censos agropecuários para os anos 1985, 1995-1996 e 2006. O estudo examina o papel do tamanho da propriedade para o acelerado crescimento da PTF agrícola observado no Brasil nos últimos 20 anos. Para tanto, os estabelecimentos agrícolas foram divididos em classes pelo tamanho e pelas suas respectivas contribuições ao crescimento da PTF nacional. Os resultados específicos para o Nordeste mostraram que a segunda menor classe de estabelecimentos (de 5 a 20 hectares) foi responsável por $18 \%$ da produção, representando $21 \%$ dos estabelecimentos da região. Esta foi a classe que mais evoluiu em eficiência técnica, de 7,75\%, bem como em relação ao crescimento da PTF (de 4,12\%). Não obstante, a maior parte das terras da região, $69 \%$ do total, é composta de grandes estabelecimentos agrícolas (de 100 a 500 hectares). Esta faixa apresentou as mais reduzidas taxas de crescimento da PTF e de ganhos de eficiência.

\section{Metodologia}

\subsection{Meta-Fronteira de Produção Estocástica}

A construção da Meta-Fronteira de Produção (MFP) se dá em dois estágios. O primeiro é dado pela construção de fronteiras estocásticas regionais. Para tanto, considere que a relação entre insumos e produtos das diversas firmas em diferentes regióes, numa dada indústria, é representada por uma fronteira estocástica regional para cada grupo de firmas. Sendo assim, para cada j-ésimo grupo, existe uma amostra de $N_{j}$ firmas, produzindo um produto a partir de vários insumos, $\mathrm{e}$ a fronteira estocástica para estes grupos é definida por:

$y_{i t}{ }^{j}=f\left(X_{i t}, \beta^{j}\right) \cdot \exp \left\{v_{i t}^{j}-u_{i t}^{j}\right\}$

Em que $y_{i t}$ é denotado como a produção observada na firma (neste caso o munícipio) $i$, no ano $t ; X_{i t}$ representa o vetor $1 x \mathrm{~K}$ de insumos ou outras variáveis explicativas associadas com a firma; $\beta^{j}$ denota o vetor $K x 1$, parâmetros desconhecidos associados ao grupo $j$. Considerou-se cada município brasileiro como uma firma, divididas em regiões, assim, tem-se $j=$ Nordeste semiárido e Nordeste não semiárido. No intuito de testar a hipótese de que as tecnologias usadas nos municípios das distintas regiões do presente estudo são as mesmas, serão estimadas fronteiras estocásticas regionais, referente às regiões, e uma com todos os municípios agrupados, desconsiderando qualquer diferenciação regional entre os municípios, de cada modelo.

A adoção dessa abordagem de fronteira estocástica tem vantagens e desvantagens. O modelo de fronteira estocástica que estima as eficiências técnicas das firmas dá-se por parâmetros testados a partir de erros padrões, além de acrescentar fatores aleatórios não negligenciáveis próprios a diversas atividades econômicas, como na agricultura (cheias, secas, dentre outras).

Deve-se ainda, salientar algumas limitações existentes na presente abordagem, como evidencia Liu et al. (2008), ao destacarem que o modelo de Fronteira Estocástica tende a ser sensível à má especificação da distribuição assumida para o termo da ineficiência, especialmente na utilização da distribuição half-normal. Já Reinhard et al. (2000) apontaram que o modelo de Fronteira Estocástica viola ocasionalmente as propriedades da função de produção ${ }^{5}$, principalmente a monotonicidade. Isso é diferente do que ocorre no modelo de DEA, em que essas condições são satisfeitas pela construção do problema de programação linear. Outro ponto passivo de críticas é a imposição de uma forma funcional, além de não possibilitar a mensuração de escores de eficiência na existência de firmas com multi-insumos e multiprodutos (Tannuri-Pianto et al., 2009).

A composição do termo do erro se dá pelos termos $v_{i t}{ }^{j} \mathrm{e} u_{i t}{ }^{j}$, sendo estes independentes entre si. O primeiro representa as variações por choques aleatórios, erros de medição etc., que são por hipótese $i i d N\left(0, \sigma_{v}{ }^{2}\right)$, ou seja, independentes e identicamente distribuídos, com distribuição normal de média zero e variância $\sigma^{2}$. Já o segundo refere-se à ineficiência técnica própria da firma, que desvia o nível de produção da firma para baixo da fronteira de produção estimada, podendo apresentar diversos tipos de distribuição, como a half-normal, como em Aigner, Lovell \& Schmidt (1977), normal truncada (Battese \& Coelli, 1995), exponencial, gama. Assume-se ainda que: $E\left(v_{i t}\right)=0$ (Média zero); $E\left(v_{i t}{ }^{2}\right)=\sigma_{v}{ }^{2}$ (homocedasticidade); $E\left(v_{i t} v_{z t}\right)=0$. para todo $\mathbf{i} \neq \mathbf{Z}$ (não

5 As principais propriedades associadas à função de produção são discutidas por Coelli et al. (2005). 
correlacionados); $E\left(u_{i t}{ }^{2}\right)=$ constante, (homocedasticidade); $E\left(u_{i t} u_{z t}\right)=0$ para todo $\mathrm{i} \neq \mathrm{Z}$, (não correlacionados).

As estimações desses parâmetros serão obtidas pelo método da máxima verossimilhança, como proposto por Battese \& Coelli (1992) ${ }^{6}$. Aigner et al. (1977) parametrizaram a função log-máxima verossimilhança e é expressa em termos da variância dos parâmetros: $\sigma^{2}=\sigma_{v}^{2}+\sigma_{u}^{2}$; $\lambda^{2}=\sigma_{u}^{2} / \sigma_{v}^{2} \geq 0$. Se $\lambda=0$, não existe o efeito de ineficiência técnica e todos os desvios da fronteira são devidos aos ruídos estocásticos. Usando essa parametrização, a função de log-máxima verossimilhança é definida pela seguinte função:

$\ln L(y \mid \beta, \sigma, \lambda)=-\frac{I}{2}\left(\frac{\pi \sigma^{2}}{2}\right)+\sum_{i=1}^{t} \ln \phi\left(\frac{\varepsilon_{i} \lambda}{\sigma}\right)-\frac{1}{2 \sigma^{2}} \sum_{i=1}^{t} \varepsilon_{l}^{2}$

Em que y é um vetor de log-produto; $\varepsilon=v_{i}-u_{i}=\ln y-x_{i}^{\prime} \beta$ é a composição do termo do erro; e $\phi(x)$ é a função de distribuição acumulada (fda) da variável aleatória com distribuição normal padrão avaliada em x.

Battese \& Corra (1977) consideraram mais convinente parametrizar a função de log-máxima verossimilhança em termos de $\sigma^{2}=\sigma_{v}^{2}+\sigma_{u}^{2}$ e $\gamma=\frac{\sigma_{u}^{2}}{\sigma^{2}}$. O parâmetro $\gamma$ está entre zero e um. Se $\gamma=0$, tem-se que todos os desvios à fronteira de produção devem-se a choques aleatórios; no entanto, quando $\gamma=1$, tem-se que todos os desvios são causados pela ineficiência técnica. As estimações desses parâmetros serão obtidas pelo método da máxima verossimilhança. $\mathrm{O}$ resultado da eficiência técnica da firma (município) especifica é dada pela relação:

$$
E T_{i t}^{j}=\frac{y_{i t}^{j}}{f\left(X_{i t}^{j} \beta_{i t}^{j}\right) \cdot \exp \left\{v_{i t}^{j}-u_{i t}^{j}\right\}}=\left\{\exp \left(-u_{i t}^{j}\right)\right\}, j=\text { semiárido, não semiárido }
$$

Para testar a hipótese de que as regiões são representadas pela mesma fronteira de produção estocástica, utiliza-se o teste da razão de verossimilhança (LR). Se a hipótese nula for rejeitada, a fronteira de produção agrupada é rejeitada e se aceita a hipótese alternativa de existência de fronteiras regionais distintas. No entanto, as ET computadas pelas fronteiras regionais estimadas não podem ser comparadas e, para tanto, adota-se o conceito de função

\footnotetext{
Poder-se-ia utilizar o modelo proposto por Battese e Coelli (1995), em que há a possibilidade de desenvolver um modelo para a explicação do termo de ineficiência técnica da firma. Houve esforços nesse sentido, principalmente para o segundo modelo. Buscou-se fatores que explicassem a ineficiência dos municípios analisados, no entanto, tal incorporação de variáveis tornou-se inviável por dois motivos: 1 - dada a indisponibilidade de dados em âmbito municipal; 2 - convergência do parâmetro $\gamma$ para as bordas, como será detalhado a seguir.
}

de meta-produção para comparação das diferenças de performances entre as duas regiões de estudo.

Parte-se da hipótese de que os munícipios estão divididos em grupos regionais e possuem diferentes conjuntos de produção, dados seus diferentes níveis de acesso aos estoques de capital físico, humano e financeiro, como nas diferentes dotações de recursos naturais, disparidade na qualidade do solo, clima, acesso a recursos energéticos, infraestrutura etc. Assim, a MF possibilita a comparação das TE das diferentes fronteiras estimadas. A função de MF de produção toma a seguinte forma:

$$
y_{i t}^{*}=f\left(X_{i t}, \beta^{*}\right) \equiv \exp \left\{X^{\prime}{ }_{i t} \beta^{*}\right\}
$$

Em que $y_{i t}^{*}$ indica o produto da meta-fronteira e $\beta^{*}$ indica o vetor de parâmetros para a MF de produção que satisfaça a seguinte restrição:

$X_{i t}^{\prime} \beta^{*} \geq X_{i t}^{\prime} \beta^{j}$, para $j=1,2 \ldots J$

Essa restrição garante que nenhuma fronteira regional seja maior que a meta-fronteira. Dessa forma, para a estimação da MFP, proposto por Battese et al. (2004) e O'Donnell et al. (2008) considera que, dado os parâmetros estimados $\hat{\beta}^{j}$ das fronteiras regionais no primeiro passo, o segundo passo para a estimação da MF se dá na estimação de $\beta^{*}$ pela solução do seguinte problema de programação linear:

$$
\begin{aligned}
& \min _{\beta} \bar{X} \beta^{*} \\
& \text { s.t. } X_{i t}^{\prime} \beta^{*} \geq X_{i t}^{\prime} \beta^{j}, \text { para } j=1,2, \ldots J, \text { para todo } i \text { e } t
\end{aligned}
$$

em que $\overline{X^{\prime}}$ é o vetor coluna das médias dos elementos dos $x_{i t}$-vetores para todas as observações no conjunto de dados. Com o problema de programação linear resolvido, tem-se o vetor $\beta^{*}$ definido, rescreve-se o produto observado da firma (município) $i$, na região $j$ no ano $t$, pela seguinte decomposição:

$$
\begin{aligned}
& y_{i t}^{j}=\exp \left(-u_{i t}^{j}\right) \times \frac{f\left(X_{i t}^{j}, \beta^{j}\right)}{f\left(X_{i t}^{j}, \beta^{*}\right)} \times f\left(X_{i t}^{j}, \beta^{*}\right) \exp \left(v_{i t}^{j}\right) \\
& M_{T R}^{j}=\frac{f\left(X_{i t}^{j}, \beta^{j}\right)}{f\left(X_{i t}^{j}, \beta^{*}\right)}, \text { onde } 0 \leq M T R_{i t}^{j} \leq 1
\end{aligned}
$$

O primeiro termo do lado direito da equação (8) representa a eficiência técnica $(\mathrm{ET})$ relativa à fronteira estocástica da j-ésima região. O segundo termo é nomeado de razão de meta-tecnologia (MTR), que representa a diferença entre a tecnologia disponível para a região $j$, 
relativa à melhor tecnologia disponível para a indústria como um todo, e exposta na equação (9). A eficiência técnica relativa à meta-fronteira estocástica é definida de uma forma análoga à equação (10):

$$
E T_{i t}^{* j}=\frac{y_{i t}^{j}}{f\left(X_{i t}^{j}, \beta^{*}\right) \exp \left(v_{i t}^{j}\right)}=T E_{i t}^{j} \times M R T_{i t}^{j}
$$

\subsection{Base de dados}

Os dados utilizados nos dois modelos baseiam-se nos censos agropecuários do IBGE de 1975 a 2006, além do sistema de contas regionais, também disponibilizadas pela plataforma do Ipeadata, em uma série temporal de 1975, 1985, 1995/1996 e 2006. Os municípios não existentes nos períodos do censo, ou que possuíam informações omitidas para alguma variável em algum dos anos de análises, foram excluídos da amostra, de forma a manter os dados em painel balanceado. Dos 1989 munícipios iniciais, a amostra final contabilizou 1268 municípios que continuaram nesta análise, sendo 740 pertencentes ao semiárido e 528 ao não semiárido.

A variável escolhida para representar o produto foi o PIB agrícola municipal, disponibilizado pelo Ipeadata e deflacionado a preços de 2000 pelo deflator implícito do PIB. Para a mensuração dos fatores de produção terra, trabalho e despesas com insumos, a coleta de dados dependeu exclusivamente dos dados disponibilizados pelos censos agropecuários. $\mathrm{O}$ fator terra foi considerado como a área total das terras plantadas com lavouras permanentes e temporárias. Não foram contabilizadas aí áreas plantadas com pastagens, por não serem consideradas como fator escasso para agropecuária, como apresenta Bragagnolo (2012).

Para medir a força de trabalho, optou-se por utilizar os dados referentes ao pessoal ocupado na agropecuária, que inclui tanto proprietários, quanto trabalhadores contratados. Já as despesas com insumos foram retiradas das despesas declaradas em fertilizantes, defensivos, sementes, mudas, energia, dentre outros. Estes valores estão deflacionados pelo deflator implícito do PIB nacional. Para a variável estoque de capital, utilizou-se o valor dos bens dos agricultores, disponibilizados pelo censo agropecuário, incluindo as construções rurais, máquinas e equipamentos. Contudo, essa variável não foi disponibilizada no censo agropecuário de 1995/1996, o que obrigou a utilização de uma proxy para este período em especial, tal como proposto por Bragagnolo (2012). O método consistiu de partir dos dados disponíveis em 1985, dos quais se acumularam os valores dos investimentos anuais até 1995, aplicando ainda uma depreciação de $6 \%$, de acordo com os valores apresentados em Gasques et al. (2012). Esta variável foi deflacionada em Reais (R\$) de 2000 pelo deflator implícito da formação bruta de capital, disponibilizado pelo Ipeadata.

Ressalta-se que os censos agropecuários do IBGE de 1975 a 2006 tiveram modificações metodológicas ao longo do tempo. No entanto, os resultados apresentados na seção seguinte corroboram com a literatura especializada, o que demostra a coerência das estimativas.

\subsection{Especificação do modelo empírico}

Para o cálculo da meta-fronteira de produção, deve-se primeiramente estimar as fronteiras regionais por fronteira estocástica. Para tanto, utiliza-se o método proposto por Aigner et al. (1977) e Meeusen \& Van Den Broeck (1977) de fronteira estocástica de produção, o qual foi aprimorado por Schimidt \& Sickles (1984) e Battese \& Coelli (1992; 1995). Estas modificações permitem estimar a fronteira estocástica com dados em painel.

Nesse sentido, inicialmente definiu-se a forma funcional da fronteira estocástica de produção. Existem diferentes formas funcionais que dão origem a diferentes modelagens econométricas na análise de fronteiras estocásticas. As formas funcionais mais comuns na literatura são a Cobb-Douglas (CD) e a Translog (TL). Ahmad \& Bravo-Ureta (1997) argumentam que a mensuração da ET é robusta para ambas as formas funcionais.

$\ln Y_{i t}{ }^{j}=\beta_{1}+\beta_{2} \ln L_{i t}{ }^{j}+\beta_{3} \ln T_{i t}{ }^{j}+\beta_{4} \ln K_{i t}{ }^{j}+\beta_{5} \ln I_{i t}{ }^{j}+\beta_{6} t^{j}+v_{i t}{ }^{j}-u_{i t}{ }^{j}$

Em que: $Y_{i t}=$ produto agropecuário do município $i$ no período $t$, da região $j ; L_{i t}=$ fator de produção terra do município $i$ no período $t$ da região $j ; T_{i t}=$ trabalho por município $i$ no período $t$ da região $j ; K_{i t}=$ estoque de capital por município $i$ no período $t$ da região $j ; I_{i t}=$ despesas com insumos por município $i$ no período $t$ da região $j$; $t=$ tendência linear da região $j ; v_{i t}=$ distúrbios aleatórios da função de produção que por hipótese iid $\sim N\left(0, \sigma^{2}\right)$; $u_{i t}=$ ineficiência técnica da produção que por hipótese pode assumir que iid $\sim H N\left(0, \sigma^{2}\right)$ ou $u_{i t} \sim i i d N^{+}\left(\mu, \sigma_{v}{ }^{2}\right)$. 


\subsection{Testes realizados}

\subsubsection{Teste da forma funcional}

O teste se dá da seguinte forma: após a obtenção dos dois modelos, encontra-se os seus respectivos valores de log-verossimilhança $(L L)$. Com estes resultados em mãos, o teste da razão de verossimilhança (LR) tem como hipótese nula $\mathrm{H}_{\mathrm{o}}$ : LL Cobb-Douglas, e hipótese alternativa, $\mathrm{H}_{1}$ : LL Translog. O LR é calculado pela seguinte equação: $\mathrm{LR}=-2\left[\ln \mathrm{LL} \mathrm{H}_{\mathrm{o}}-\ln \mathrm{LL} \mathrm{H}_{1}\right] \sim \chi^{2}$; no qual LL é o log-verossimilhança estimado para cada forma funcional. Utilizando os valores críticos mostrados por Kodde \& Palm (1986), falha-se em rejeitar $\mathrm{H}_{\mathrm{o}^{\prime}}$ se LR < T KP (Tabela de Kodde \& Palm, 1986).

\subsubsection{Efeito da ineficiência técnica na função de produção}

Testa-se a inexistência da ineficiência técnica, ou seja, se de fato o modelo capta a ineficiência da firma. Neste caso, toma-se o valor da log-verossimilhança do modelo estimado sem esta variável. Pelo método de mínimos quadrados ordinários, aplica-se novamente o teste de verossimilhança generalizada, comparando-se ao valor crítico da tabela de Kodde \& Palm (1986). Assim, o teste da razão de verossimilhança (LR) tem como hipótese nula, $\mathrm{H}_{\mathrm{o}}$ : Modelo de mínimos quadrados ordinários (inexistência de ineficiência técnica, $\gamma=0$ ) e hipótese alternativa, $\mathrm{H}_{1}$ : LL Hipótese alternativa: a ineficiência técnica deve ser considerada no modelo, $\gamma \neq 0$. O LR é calculado pela seguinte equação: $\mathrm{LR}=-2\left[\ln \mathrm{LL} \mathrm{H}_{\mathrm{o}}-\ln \mathrm{LL} \mathrm{H}_{1}\right] \sim \chi^{2}$; no qual LL é o log-verossimilhança estimado para cada forma funcional. Utilizando os valores críticos mostrados por Kodde \& Palm (1986), falha-se em rejeitar $\mathrm{H}_{\mathrm{o}}$, se LR < T KP (Tabela de Kodde \& Palm, 1986).

\subsubsection{Distribuição do Termo da Ineficiência}

Este teste é utilizado para indicar qual a distribuição do termo da eficiência é a mais indicada para o modelo. Primeiro, estima-se uma fronteira estocástica com o termo do erro com distribuição half-normal $\left(\mathrm{H}_{\mathrm{o}}\right) \mathrm{e}$, em seguida, com distribuição truncada normal $\left(\mathrm{H}_{1}\right)$, confrontando-as pelo teste de máxima verossimilhança. O LR é calculado pela seguinte equação: $\mathrm{LR}=-2\left[\ln \mathrm{LL} \mathrm{H}_{\mathrm{o}}-\ln \mathrm{LL} \mathrm{H}_{1}\right]$ $\sim \chi^{2}$; no qual LL é o log-verossimilhança estimado para cada forma funcional. Utilizando os valores críticos mostrados por Kodde \& Palm (1986), falha-se em rejeitar $\mathrm{H}_{\mathrm{o}}$, se LR < T KP (Tabela de Kodde \& Palm, 1986).

\subsubsection{Ausência de progresso técnico}

Neste teste, considera-se que os coeficientes correspondentes às variáveis relacionadas ao tempo na função Cobb-Douglas são iguais a zero ou não. O teste da razão de verossimilhança (LR) tem como hipótese nula, $\mathrm{H}_{\mathrm{o}}$ : LL $\beta_{6}=0$, e hipótese alternativa, $\mathrm{H}_{1}$ : LL $\beta_{6} \neq 0$. O LR é calculado pela seguinte equação: $\mathrm{LR}=-2\left[\ln \mathrm{LL} \mathrm{H}_{\mathrm{o}}-\ln \mathrm{LL} \mathrm{H}_{1}\right] \sim \chi^{2}$; no qual LL é o log-verossimilhança estimado para cada forma funcional. Utilizando os valores críticos mostrados por Kodde \& Palm (1986), falha-se em rejeitar $\mathrm{H}_{0}$ se $\mathrm{LR}<\mathrm{T}$ KP (Tabela de Kodde \& Palm, 1986).

\subsubsection{Existência de duas fronteiras regionais}

Para testar a hipótese de que as duas regiões podem ser representadas pela mesma fronteira de produção estocástica utiliza-se o teste da razão de verossimilhança (LR), tomando como hipótese nula, $\mathrm{H}_{\mathrm{o}}$ : LL da fronteira agrupada e hipótese alternativa, $\mathrm{H}_{1}$ : é a soma dos valores de $L L$ das fronteiras regionais referente às demais regiões. Novamente, o LR é calculado pela seguinte equação: $\mathrm{LR}=-2\left[\ln \mathrm{LL} \mathrm{H}_{\mathrm{o}}-\ln \mathrm{LL} \mathrm{H}_{1}\right] \sim \chi^{2}$; no qual LL é o log-verossimilhança estimado para cada forma funcional. Utilizando os valores críticos mostrados por Kodde \& Palm (1986), falha-se em rejeitar $\mathrm{H}_{\mathrm{o}}$, se $\mathrm{LR}<\mathrm{T} \mathrm{KP}$ (Tabela de Kodde \& Palm, 1986). Se a hipótese nula for rejeitada, a fronteira de produção agrupada é rejeitada e se aceita a hipótese alternativa de existência de fronteiras regionais distintas.

\section{Resultados e discussões}

\subsection{Estatísticas descritivas}

As estatísticas descritivas estão expostas na Tabela 2. Conforme os dados, no que tange às médias, aos desvios padrão e às amplitudes, observam-se poucas variações significativas entre as regiões estudadas, o semiárido e os demais municípios. No âmbito inter-regional, as duas regiões não têm grandes disparidades.

O produto agrícola do semiárido é, em média, ligeiramente maior do que no não semiárido. Todavia, 
Tabela 2. Estatísticas descritiva

\begin{tabular}{cccccc}
\hline Variáveis & Região & Média & Desvio padrão & Mínimo & Máximo \\
\hline \multirow{2}{*}{ Y (R\$ milhares) } & Semiárido & 10502,35 & 16075,65 & 115,3 & 216760,36 \\
& Não semiárido & 10499,70 & 20090,09 & 31,00 & 360200,00 \\
L (hectare) & Semiárido & 8305,93 & 10646,28 & 60,22 & 265468,37 \\
T (pessoas & Não semiárido & 8302,59 & 10645,91 & 1,76 & 176100,00 \\
ocupadas) & Semiárido & 6170,72 & 5636,13 & 37,00 & 39480,00 \\
K (R\$ milhares) & Não semiárido & 6166,34 & 5630,59 & 18,00 & 67190,00 \\
& Semiárido & 156976,98 & 389728,99 & 574,00 & 9905797,00 \\
I (R\$ milhares) & Não semiárido & 156972,82 & 389727,91 & 285 & 7813000,00 \\
& Semiárido & 5684,58 & 16197,33 & 53,20 & 22142,65 \\
\hline
\end{tabular}

Fonte: Elaboração dos autores a partir dos resultados da pesquisa.

apresenta menor desvio padrão e menor amplitude, representando que os municípios do semiárido são mais homogêneos que os do não semiárido. No que tange aos fatores de produção, o semiárido consome, em média, ligeiramente mais em todos os insumos.

O não semiárido gasta, em média, quase a mesma quantidade do fator capital médio ligeiramente mais elevado. Isso significa que o semiárido possui um quantitativo superior de maquinaria, veículos e construções. No que se concerne às despesas com insumos, o não semiárido e o semiárido consomem, em média, a mesma quantidade de energia, de mudas, de serviços veterinários, dentre outros. Essa ligeira superioridade deve ser relativizada, já que o quantitativo de municípios existentes no semiárido brasileiro é bem superior ao da soma dos municípios pertencentes ao não semiárido (740 pertencentes ao semiárido e 528 ao não semiárido).

\subsection{Testes de hipóteses}

Após as estimações das fronteiras regionais, a partir dos respectivos valores de log máxima verossimilhança das estimações de cada fronteira, os valores $\lambda$, foram realizados os respectivos testes e que são apresentados na Tabela 3, a saber: de forma funcional para escolha do melhor modelo; o de ineficiência técnica; o teste de ausência de progresso técnico; como também o de existência de fronteiras regionais. As estimações ${ }^{7}$ da forma funcional translog apresentaram, para pelo menos uma das fronteiras regionais, indicativos de não haver

As estimações estão disponíveis para consulta com os autores. ineficiência ou de má especificação do modelo, já que os resultados indicavam que o termo do erro estava a violar a hipótese de normalidade de sua distribuição. Desta forma, optou-se pela forma funcional Cobb-Douglas, pois mostrou-se a mais adequada para as três fronteiras estimadas, não havendo indícios de má especificação.

Escolhida a forma funcional, realizou-se o teste de ausência de ineficiência técnica ao modelo, caso $\gamma=0$ (não se deve utilizar o Modelo de Fronteira Estocástica, ou seja, o modelo não capta o efeito ineficiência). Nesse caso, é indicada a utilização do método de mínimos quadrados ordinários. Têm-se os resultados do teste para as três fronteiras, que superam seus respectivos valores críticos e significância de 5\% na tabela de Kodde \& Palm (1986), dados seus graus de liberdade. Portanto, rejeita-se $H_{0}$ e se aceita a hipótese $H_{1}$, confirmando a presença de progresso técnico para as três fronteiras apresentadas.

Em seguida, testou-se qual a melhor distribuição estatística para o termo da ineficiência. Estimou-se uma fronteira para cada região, uma com distribuição half-normal, enquanto outra com distribuição truncada normal e, em seguida, confrontando-as pelo LR teste. Os resultados apontaram que a melhor escolha é a utilização da distribuição truncada normal. Dessa forma, salvaguarda o modelo dos problemas de má especificação apresentada por Liu et al. (2008), quando se assume a distribuição half-normal para o termo da ineficiência. Posteriormente, testou-se a ausência de progresso técnico. De acordo com o teste descrito anteriormente, estimou-se o modelo na forma funcional Cobb-Douglas e na forma com ausência de progresso técnico. De acordo com os resultados, rejeitou-se a hipótese nula e aceitou-se a 
Tabela 3. Prova de razão verossimilhança dos parâmetros das fronteiras de produção

\begin{tabular}{|c|c|c|c|c|c|c|}
\hline Prova & Região & Hipótese Nula & $\begin{array}{l}\text { Graus de } \\
\text { Liberdade }\end{array}$ & $\Lambda$ & $\begin{array}{l}\text { Valor } \\
\text { crítico }\end{array}$ & Decisão \\
\hline \multirow{3}{*}{$\begin{array}{l}\text { Inexistência de Eficiência } \\
\text { Técnica }\end{array}$} & Fronteira agrupada & $H_{0}: \gamma=0$ & 1,00 & 715,75 & 2,7 & Rejeita $H_{0}$ \\
\hline & Fronteira semiárido & $H_{0}: \gamma=0$ & 1,00 & 430,79 & 2,7 & Rejeita $H_{0}$ \\
\hline & $\begin{array}{l}\text { Fronteira não } \\
\text { semiárido }\end{array}$ & $H_{0}: \gamma=0$ & 1,00 & 229,32 & 2,7 & Rejeita $H_{0}$ \\
\hline \multirow{3}{*}{$\begin{array}{l}\text { Distribuição do Termo da } \\
\text { Ineficiência }\end{array}$} & Fronteira agrupada & $H_{0}:$ Half-Normal & 1,00 & 145,93 & 2,7 & Rejeita $H_{0}$ \\
\hline & Fronteira semiárido & $H_{0}:$ Half-Normal & 1,00 & 129,07 & 2,7 & Rejeita $H_{0}$ \\
\hline & $\begin{array}{l}\text { Fronteira não } \\
\text { semiárido }\end{array}$ & $H_{0}:$ Half-Normal & 1,00 & 31,623 & 2,7 & Rejeita $H_{0}$ \\
\hline \multirow{3}{*}{$\begin{array}{l}\text { Ausência de progresso } \\
\text { técnico }\end{array}$} & Fronteira agrupada & $H_{0}: \beta_{6}=0$ & 1,00 & 178,19 & 2,7 & Rejeita $H_{0}$ \\
\hline & Fronteira semiárido & $H_{0}: \beta_{6}=0$ & 1,00 & 148,07 & 2,7 & Rejeita $H_{0}$ \\
\hline & $\begin{array}{l}\text { Fronteira não } \\
\text { semiárido }\end{array}$ & $H_{0}: \beta_{6}=0$ & 1,00 & 25,55 & 2,7 & Rejeita $H_{0}$ \\
\hline $\begin{array}{l}\text { Existência de duas } \\
\text { fronteiras regionais }\end{array}$ & Não se aplica & $\begin{array}{c}H_{0}: \text { Fronteira } \\
\text { agrupada }\end{array}$ & 8,00 & 178,00 & 14,8 & Rejeita $H_{0}$ \\
\hline
\end{tabular}

Fonte: Elaboração dos autores a partir dos resultados da pesquisa. $\lambda$ : prova estatística da razão de máximo verossimilhança em que $\lambda=-2\left[L L\left(H_{0}\right)-L L\left(H_{1}\right)\right] \sim \chi^{2}$, com graus de liberdade iguais ao número de restrições independentes.

hipótese alternativa, validando o uso do modelo de fronteira estocástica.

Por fim, realizou-se o teste para validar a existência da diferenciação entre as possibilidades produtivas entre as duas regiões ou não. Conforme os resultados encontrados, aceita-se a hipótese alternativa, validando que os municípios pertencentes ao semiárido ou ao não semiárido empregam diferentes tecnologias de produção. Logo, pode-se utilizar o quadro teórico da meta-fronteira de produção, a fim de comparar o desempenho de eficiências entre as diferentes regiões.

\subsection{Estimação das fronteiras de produção}

Os resultados das estimações estão presentes na Tabela 4. Vale salientar que, para todas as fronteiras, foi realizado o teste de hipótese pelo teste razão de verossimilhança generalizada, no intuito de verificar se o termo do erro correspondente à ineficiência seria inexistente. Rejeitou-se a hipótese nula de inexistência da eficiência técnica em todas as fronteiras a um nível de significância de $5 \%$.

Nas três fronteiras estimadas, todos os parâmetros são altamente significativos a um nível de $5 \%$, implicando em função de produção bem-comportada, com quase todos os parâmetros com sinais esperados. Chamou atenção o quão foram semelhantes as duas fronteiras regionais, sendo as mesmas variáveis, impactando positivamente na produção agrícola em ambas as regiões, ao mesmo tempo que se observa a composição heterogênea dos impactos de cada fator de produção entre as regiões. Pode-se concluir que políticas públicas, como o FNE, que proporcionem investimentos e compras de equipamentos, melhoria de acesso a insumos modernos são fundamentais ao estímulo da produção agropecuária nordestina.

Os fatores de produção terra, trabalho e capital impactam positivamente no produto agrícola em ambas as fronteiras regionais, como esperado. Existem diferenças regionais na magnitude desses impactos na produção. A influência do fator capital (K) e do fator Terra (L) são superiores no semiárido, o que sinaliza que investimentos em tecnologia, bem como práticas de convivência com o semiárido e suas condições são essenciais para transpor os desafios climáticos e produtivos da região.

O fator trabalho impacta positivamente na produção em ambas as fronteiras regionais, apontando o quão importante é o investimento em educação para o Nordeste como um todo, no sentido de potencializar esses ganhos. Esta assertiva é confirmada por Araújo \& Santos (2009), que perceberam que o Nordeste necessita de melhoras na educação para alcançar o desempenho nacional. É perceptível que o fator capital influencia positivamente na produção agropecuária e é ligeiramente maior no semiárido, corroborando com os resultados de Gasques (2014). Dessa forma, a modernização do maquinário, que substitui tratores de menor potência pelo uso de 
Tabela 4. Fronteiras estocásticas estimadas para o Nordeste, semiárido e não semiárido

\begin{tabular}{|c|c|c|c|c|}
\hline Variáveis & Parâmetros & Nordeste & não semiárido & semiárido \\
\hline \multirow[t]{2}{*}{ (intercepto) } & $\beta_{1}$ & $1,6^{* * *}$ & $1,70^{* * *}$ & $1,46^{* * *}$ \\
\hline & & $(0,11)$ & $(0,17)$ & $(0,14)$ \\
\hline \multirow{2}{*}{$L_{i t}$} & $\beta_{2}$ & $0,15^{* * *}$ & $0,13^{* * *}$ & $0,19^{* * *}$ \\
\hline & & $(0,01)$ & $(0,02)$ & $(0,02)$ \\
\hline \multirow[t]{2}{*}{$T_{i t}$} & $\beta_{3}$ & $0,23^{* * *}$ & $0,28^{* * *}$ & $0,18^{* * *}$ \\
\hline & & $(0,01)$ & $(0,02)$ & $(0,02)$ \\
\hline \multirow[t]{2}{*}{$K_{i t}$} & $\beta_{4}$ & $0,16^{* * *}$ & $0,13^{* * *}$ & $0,19^{* * *}$ \\
\hline & & $(0,01)$ & $(0,01)$ & $(0,01)$ \\
\hline \multirow[t]{2}{*}{$I_{i t}$} & $\beta_{5}$ & $0,3^{* * *}$ & $0,3^{* * *}$ & $0,29^{* * *}$ \\
\hline & & $(0,01)$ & $(0,01)$ & $(0,01)$ \\
\hline \multirow[t]{12}{*}{$T$} & $\beta_{6}$ & $0,11^{* * *}$ & $0,13^{* * *}$ & $0,08^{* * *}$ \\
\hline & & $(0,01)$ & $(0,02)$ & $(0,02)$ \\
\hline & $\sigma^{2}$ & $1,28^{* * *}$ & $1,14^{* * *}$ & $1,17^{* * *}$ \\
\hline & & $(0,08)$ & $(0,05)$ & $(0,09)$ \\
\hline & $\Gamma$ & $0,79^{* * *}$ & $0,73^{* * *}$ & $0,79^{* * *}$ \\
\hline & & $(0,01)$ & $(0,03)$ & $(0,02)$ \\
\hline & $\sigma_{\mathrm{u}}^{2}$ & $1,01^{* * *}$ & $0,84^{* * *}$ & $0,93^{* * *}$ \\
\hline & & $(0,08)$ & $(0,11)$ & $(0,1)$ \\
\hline & $\sigma_{\mathrm{v}}^{2}$ & $0,27^{* * *}$ & $0,30^{* * * *}$ & $0,24^{* * *}$ \\
\hline & & $(0,01)$ & $(0,01)$ & $(0,0)$ \\
\hline & ET média & 0,67 & 0,69 & 0,68 \\
\hline & Log-verossimilhança & $-4.546,434$ & $-1.982,29$ & $-2.497,46$ \\
\hline
\end{tabular}

Fonte: Elaboração dos autores a partir dos resultados da pesquisa. Em que: $\gamma=\frac{\sigma^{2} u}{\sigma_{v}^{2}+\sigma_{u}^{2}}$. ${ }^{* *}$ significante a um nível de $5 \% .{ }^{*}$ A codificação para a significância dos coeficientes estimados.

veículos de médio e grande porte, acarretaria ganhos na produção do setor. Daí a importância da política de juros subsidiados proporcionados pelo FNE, com enfoque no semiárido, na ampliação do estoque de capital.

O fator de produção terra influencia positivamente a produção em ambas as fronteiras regionais, sendo mais significativa no não semiárido nordestino. O trabalho de Helfand et al. (2015) discute a estrutura fundiária do Nordeste. Segundo os autores, os estabelecimentos mais eficientes e produtivos da região Nordeste são as pequenas propriedades. Todavia, $69 \%$ das terras estão em propriedade dos estabelecimentos de grande porte, os menos produtivos da região. A incorporação de novas áreas para os produtores mais eficientes e produtivos da região é bastante restrita, podendo caracterizar-se como um entrave ao aumento da produtividade/eficiência via aumento da área cultivada desses pequenos produtores eficientes.

As variáveis despesas com insumos e a tendência temporal têm relação positiva com a produção em ambas as fronteiras regionais. No que tange a despesas com insumos, há uma indicação de causalidades perante as condições próprias da região, que tornam estes gastos fundamentais ao processo produtivo, pois as três fronteiras apresentaram a mesma magnitude de impacto, sendo o fator que mais impacta no processo produtivo. Nesse ponto, quanto maiores são os gastos com insumos, maiores seriam o retorno produtivo, ou seja, o uso intensivo de insumo representa aumento de produção.

Salienta-se que este é o fato de produção que mais impacta na produção, tanto para o semiárido quanto para o não semiárido. Isso se dá pelo fato de a região demandar intensamente adubos, defensivos, gastos com energia e água para manter uma área irrigada, dadas as características próprias da região, principalmente no que tange ao semiárido do Nordeste do Brasili ${ }^{8}$. Vicente (2004) aponta como possíveis causas para o baixo dinamismo da

8 No Nordeste, há o predomínio de solos do tipo litossolos, bruno não cálcicos, lítólicos eutróficos, dentre outros que possuem baixa fertilidade natural, condição que se agrava dada a baixa pluviosidade da região. Nesses tipos de solo, mesmo com investimento em insumos, os retornos em produtividade estão abaixo dos esperados, quando comparados com solos com boa aptidão agrícola. 
Tabela 5. Estatística descritiva e distribuição de frequência das EF (\%) e MTR (\%) estimadas

\begin{tabular}{ccccc}
\hline Estatísticas & $\boldsymbol{E T}_{\text {Semiárido }}$ & $\boldsymbol{M T R}_{\text {Semiárido }}$ & $\boldsymbol{E T}_{\text {semiárido }}^{*}$ & $\boldsymbol{E T}_{\text {nâo semiárido }}=\boldsymbol{E T _ { \text { não semiárido } } ^ { * }}$ \\
\hline Média & 67,78 & 73,98 & 50,22 & 69,52 \\
Desvio padrão & 18,25 & 4,23 & 14,02 & 17,46 \\
Mínimo & 4,20 & 59,05 & 3,20 & 4,12 \\
Máximo & 98,61 & 91,92 & 83,53 & 26,6 \\
Níveis & & & & \\
$<0.6$ & 30,4 & 0,1 & 73,2 & 16,6 \\
$0,6-0,7$ & 17,3 & 18,9 & 22,5 & 23,8 \\
$0,7-0,8$ & 22,7 & 74,4 & 4,2 & 24,9 \\
$0,8-0,9$ & 21,3 & 6,6 & 0,1 & 8,2 \\
$0,9-1$ & 8,3 & 0,1 & 0,0 & \\
\hline
\end{tabular}

Fonte: Elaboração dos autores a partir dos resultados da pesquisa.

região a infraestrutura ineficiente e o limitado acesso ao crédito na região, inviabilizando a aquisição de insumos modernos, podendo estar aí a importância do FNE, para reversão do presente quadro.

No que consta sobre a mudança técnica, pôde-se dividir esse efeito em dois: a tendência linear, que aponta o progresso técnico, e o efeito tempo. A variável tendência linear foi significativa para todas as fronteiras e indica um avanço técnico do setor agrícola da região no decorrer da série histórica. Ainda se nota que o progresso técnico foi mais intenso no não semiárido, quando se comparado ao semiárido.

A média da eficiência técnica varia entre as fronteiras regionais. O não semiárido apresenta ET média de $69 \%$ e o semiárido possui o escore médio de eficiência de $68 \%$. Esta diferença não implica que os munícipios do semiárido possuem desempenhos piores que os do não semiárido, pois a comparação das duas fronteiras só fará sentindo se elas não compartilharem da mesma tecnologia de produção, representada pela meta-fronteira de produção. No entanto, se as duas regiões comungam da mesma tecnologia, deve-se usar o resultado dos munícipios agrupados do Nordeste.

\subsection{Análise das eficiências técnicas e razão de meta-fronteira}

Os parâmetros encontrados para a meta-fronteira correspondem aos apresentados na fronteira regional dos municípios situados no não semiárido, estimada pelo modelo de fronteira estocástica. Este resultado indica que a tecnologia de produção empregada na agropecuária nestes municípios é mais avançada que na região semiárida. $\mathrm{O}$ resultado aponta que a região não semiárida define tecnologia potencial disponível para todos os munícipios do Nordeste.

A razão de meta-tecnologia (MTR), definida na equação (9), é para todos os municípios do não semiárido igual a 1, pois eles situam-se na meta-fronteira de produção. A MTR média dos municípios do semiárido é por volta de $74 \%$ com um desvio padrão de $4 \%$, como pode-se observar na Tabela 5 . Esse resultado indica que, em média, os municípios do semiárido produzem no máximo $74 \%$ do que pode ser produzido na região não semiárida ao utilizar os mesmos fatores de produção e tecnologias disponíveis, podendo variar $4 \%$ para mais ou para menos.

Já a ET referente à meta-fronteira de produção é chamada de $\mathrm{ET}^{*}$ e foi estimada para todos os municípios do semiárido de acordo com a equação (10). Os munícipios do não semiárido, por serem pertencentes à meta-fronteira de produção, têm a sua $\mathrm{ET}^{*}$ equivalente à calculada sobre a fronteira regional. A Tabela 5 resume os resultados referentes às eficiências e à MRT. O semiárido apresenta $\mathrm{ET}^{*}$ média de 50,22\%, tendo como referência à $\mathrm{MF}$ entre 1975 e 2006. O município mais ineficiente da região possui escore de $\mathrm{ET}^{*}$ de 3,2\% (Monteiro - PB), enquanto o município mais eficiente (Petrolina - PE) tem escore de $83,53 \%{ }^{9}$. Já a região não semiárida tem escore de $\mathrm{ET}^{*}$ média de 69,52\%, superior à outra região de análise, onde a sua amplitude é tão grande quanto a apresentada no semiárido, com valor mínimo de 4,12\% e máximo de $98,1 \%$.

9 Os escores de eficiências de todos os municípios da amostra final estão com os autores, disponíveis para consulta. 
Ao comparar as distribuições de frequências das $E T_{\text {não semiárido }}^{*}$ e $E T_{\text {semiárido, }}^{*}$ ainda na Tabela 5, percebe-se que todas as observações no semiárido encontram-se praticamente entre 0,0 e 0,8 , enquanto no não semiárido se distribuem de maneira mais homogênea quando comparada ao semiárido. Essas conclusões não implicam que a situação do não semiárido seja das melhores. Nesta região, 42,76\% dos municípios apresentam escores inferiores a $70 \%$, tornando ainda mais grave a situação do Nordeste, tendo em vista a dependência das camadas mais pobres da população com a atividade agropecuária.

Quando se agrupam os municípios em estados, percebe-se uma uniformização da MRT no período de análise, como disposto na Tabela 6. Ao comparar as ETs* percebe-se que o semiárido sergipano apresenta a pior performance $(46,7 \%)$, acompanhado pelos semiáridos potiguar $(47,7 \%)$ e piauiense $(48,5 \%)$. Os maiores escores são apresentados pelo semiárido pernambucano (54,1\%), acompanhado do baiano $(52,3 \%)$ e cearense $(51,3 \%)$.

Tabela 6. Amostras sub-regionais das ET (\%) e MRT (\%) médias

\begin{tabular}{|c|c|c|c|}
\hline Região semiárida (1975-2006) & ET & MRT & ET $^{*}$ \\
\hline Alagoas & 66,5 & 74,0 & 49,2 \\
\hline Bahia & 70,6 & 74,1 & 52,3 \\
\hline Ceará & 69,3 & 74,0 & 51,3 \\
\hline Minas Gerais & 68,3 & 74,0 & 50,5 \\
\hline Paraíba & 67,5 & 74,0 & 50,0 \\
\hline Pernambuco & 72,8 & 74,3 & 54,1 \\
\hline Piauí & 65,3 & 74,2 & 48,5 \\
\hline Rio Grande do Norte & 64,3 & 74,0 & 47,7 \\
\hline Sergipe & 63,2 & 73,8 & 46,7 \\
\hline Região não semiárida (1975-2006) & & $\mathrm{ET}=\mathrm{ET}^{*}$ & \\
\hline Alagoas & & 67,8 & \\
\hline Bahia & & 72,0 & \\
\hline Ceará & & 71,0 & \\
\hline Espírito Santo & & 70,9 & \\
\hline Maranhão & & 70,3 & \\
\hline Minas Gerais & & 68,9 & \\
\hline Paraíba & & 68,4 & \\
\hline Pernambuco & & 73,8 & \\
\hline Piauí & & 66,5 & \\
\hline Rio Grande do Norte & & 66,1 & \\
\hline Sergipe & & 65,4 & \\
\hline
\end{tabular}

Fonte: Elaboração dos autores a partir dos resultados da pesquisa.
As melhores práticas observadas no não semiárido estão em Pernambuco (73,8\%), Bahia (72\%) e Ceará (71\%). O não semiárido delimitado pelo estado com o menor escore é Sergipe, o que mostra o quão problemático é a agropecuária do estado, seguido pelos não semiáridos potiguar $(30,5 \%)$ e piauiense $(31,3 \%)$ novamente, tornando estes estados com as piores práticas nos dois estratos.

Percebe-se que a opção de manter o norte de Minas Gerais e do Espirito Santo na amostra de municípios pertencentes à cartografia do Nordeste considerada pela Sudene foi acertada. Identifica-se a homogeneidade destas regiões com seu entorno, podendo-se inferir que, mesmo pertencendo a estados mais desenvolvidos, possuidores de maior arrecadação que os estados do Nordeste e, consequentemente, maior capacidade de ofertar bens e serviços públicos, ainda assim, não são capazes de diferenciar seus municípios das condições no que tange à eficiência produtiva. Esse resultado ratifica as conclusões de Irffi (2015).

É importante destacar que esses dados não captam a pungente produção de grãos que vem se instalando no cerrado do Piauí, dado o horizonte temporal utilizado, o que pode não captar as transformações recentes que estão ocorrendo no estado, bem como seus impactos na eficiência técnica de seus municípios. Essa ressalva dá-se pela base produtiva, intensiva em capital e tecnologia, que essa ocupação vem empregando na região do Matopiba (Buainain; Garcia, 2016; Vieira Filho, 2016).

\section{Conclusões}

O objetivo deste estudo foi analisar as diferenças tecnológicas da agropecuária entre os municípios situados no semiárido e no não semiárido do Nordeste brasileiro. Inicialmente, definiu-se a área de atuação da Sudene e não apenas o Nordeste geográfico brasileiro, definido pelo IBGE, no intuito de melhor representar os dois grupos regionais. $\mathrm{O}$ modelo utilizado foi de meta-fronteira proposto por Battese et al. (2004) e O'Donnell et al. (2008) que possibilitou as estimações das eficiências técnicas (ET e ET*) e da razão de meta-fronteira (MRT) dos municípios inseridos no semiárido e não semiárido nordestino. Para tanto, foram utilizados dados do Censo Agropecuário e das Contas Nacionais, para os anos de 1975, 1985, 1995/1996 e 2006. 
A partir desses procedimentos metodológicos, o resultado do modelo econométrico demonstrou que a assertiva assumida como hipótese deste estudo mostrou-se verdadeira: que o semiárido e o não semiárido nordestino não compartilham da mesma tecnologia de produção. Pelas fronteiras de produção estimado, fica evidente que as políticas públicas voltadas para agropecuária do semiárido nordestino, mais distantes da fronteira tecnológica (Meta-Fronteira de Produção) observada para o Nordeste, são fundamentais para a ruptura desse gap. Os formuladores de políticas públicas poderiam levar em conta a importância do investimento em qualificação da mão de obra, a necessidade de direcionamento do crédito subsidiado, disponibilizados pelo FNE para o aumento e modernização do estoque de capital e a aquisição de insumos modernos para ambas regiões, levando em conta a superação de gargalos logísticos que impactam na competividade da agropecuária praticada na região central do Brasil.

Outro ponto importante é fortalecer o papel das instituições de Assistência Técnica e de Extensão Rural (Ater), que estão desmanteladas na maioria dos estados do Nordeste, além de melhorar os níveis de escolaridade da população e os serviços de extensão rural com capilaridade local. Pela diferenciação latente que existe na região Nordeste, buscou-se atentar à análise da questão da produção agrícola, a saber, como que as diferenças regionais próprias do semiárido nordestino impactam nas práticas agropecuárias locais. Os resultados constataram que os municípios situados no semiárido defrontam-se com diferentes combinações factíveis de insumo-produto, bem menos eficientes do observado no Nordeste não semiárido.

Constatou-se que os problemas no setor agrícola do Nordeste, identificados extensamente pela literatura acadêmica como um todo, impactam de maneira ainda mais severa o semiárido nordestino. Numa análise em âmbito estadual, os municípios situados no Espírito Santo não se sobressaíram quando comparados aos demais agrupamentos estaduais dos municípios situados no não semiárido. O mesmo observou-se para o Norte de Minas Gerais, não se destacando dos demais, mesmo pertencendo a um estado com um maior grau de desenvolvimento. Os estados que apresentaram as piores práticas para ambas as regiões do Nordeste foram Piauí, Paraíba, Rio Grande do Norte e Sergipe.
Os resultados indicaram que, quando comparadas as duas regiões, o não semiárido possui a maior eficiência técnica média com referência à meta-fronteira $(58 \%$ contra $35 \%$ do semiárido). Este resultado sugere que, mesmo lançando mão de todas as possibilidades produtivas da região Nordeste, o não semiárido tem uma prática bem aquém da sua potencialidade ideal, pois estão utilizando fatores de produção com alto grau de ineficiência.

Uma limitação do presente estudo deve-se às mudanças metodológicas ocorridas na aferição dos censos agropecuários. Embora o período de análise compreenda 36 anos, foram utilizados apenas quatro censos, numa periodicidade de 10 anos, logo, uma subestimação dos valores, como no caso do censo de 1995/1996, pode prejudicar a qualidade das estimações. Assim, tem-se que os resultados foram coerentes com os encontrados na literatura, o que indica que o presente estudo, mesmo com todas suas limitações, consegue explicar os fatos estilizados apontados.

\section{Referências}

Ahmad, M., \& Bravo-Ureta, B. E. (1997). Technical efficiency measures for dairy farms using panel data: a comparison of alternative model specifications. Journal of Productivity Analysis, 7, 399-415.

Aigner, D. J., Lovell, C. A. K., \& Schmidt, P. (1977). Formulation and estimation of stochastic frontier production functions models. Journal of Econometrics, 6, 21-37.

Alves, E., \& Rocha, D. P. (2010). Ganhar tempo é possível? In J. G. Gasques, J. E. R. Vieira Filho \& Z. Navarro (Orgs.). A agricultura brasileira: desempenho, desafios e perspectivas (pp. 275-290). Brasília: Ipea.

Araújo, T. B., \& Santos, V. (2009). Desigualdades regionais e nordeste em formação econômica do Brasil. In T. Araújo, S. Vianna, \& J. Macambira (Orgs.). 50 anos de formação econômica do Brasil: ensaios sobre a obra clássica de Celso Furtado. Rio de Janeiro: Ipea.

Barbieri, A. F., Domingues, E., Queiroz, B. L., Ruiz, R. M., Rigotti, J. I., Carvalho, J. A. M., \& Resende, M. F. (2010). Climate change and population migration in Brazil's Northeast: scenarios for 2025-2050. Population and Environment, 31(5), 344-370.

Battese, G. E., \& Coelli, T. J. (1992). Frontier production functions, technical efficiency and panel data: with application to paddy farmers in India. Journal of Productivity Analysis, 3, 153-169. 
Battese, G. E., \& Coelli, T. J. (1995). A model for technical inefficiency effects in stochastic frontier production functions for panel data. Empirical Economics, 20, 325-332.

Battese, G. E., \& Corra, G. S. (1977). Estimation of a production frontier model: with aplication to the pastoral zone of eastern Australia. Australian Journal of Agricultural Economics, 21(3), 169-179.

Battese, G. E., Rao, D. S. P., \& O’Donnell, C. J. (2004). A metafrontier production function for estimation of technical efficiencies and technology gaps for firms operating under different technologies. Journal of Productivity Analysis, 21, 91-103.

Bragagnolo, C. (2012). Produtividade, crescimento e ciclos econômicos na agricultura brasileira (Tese de doutorado). Escola Superior de Agricultura Luiz de Queiroz, São Paulo.

Buainain, A. M., \& Garcia, J. R. (2016). Crescimento da agricultura no Cerrado nordestino: fatores condicionantes, limites e resultados socioeconômicos. In J. E. R. Vieira Filho \& J. G. Gasques. Agricultura, transformação produtiva e sustentabilidade (pp. 109-142). Brasília: IPEA.

Coelli, T. J., Rao, D. S. P., \& Battese, G. E. (2005). An introduction to efficiency and productivity analysis. Kluwer Academic Publishers.

Fornazier, A., \& Vieira Filho, J. E. R. (2012). Heterogeneidade estrutural no setor agropecuário brasileiro: evidências a partir do censo agropecuário de 2006 (Texto para Discussão, 1708). Brasília: Ipea.

Fuglie, K. O., \& Wang, S. L. (2016). New evidence points to robust but uneven productivity growth in global agriculture. Amber Waves Feature, Economic Research Service, U.S. Department of Agriculture. Recuperado em 12 de novembro de 2016, de https:/ / goo.gl/UHXpdN

Gasques, J. G. (2014). Total factor productivity in agriculture: Brazil and selected States - Productivity note. Brasília: Ministério da Agriculture, Pecuária e Abastecimento.

Gasques, J., Bastos, E. T., Valdes, C., \& Bacchi, M. (2012). Total factor productivity in Brazilian agriculture. In K. O. Fuglie, S. L. Wang \& V. E. Ball (Orgs.), Productivity growth in agriculture: an international perspective (pp. 145-162). Oxfordshire: CAB International.

Hayami, Y. (1969). Sources of agricultural productivity gap among selected countries. American Journal of Agricultural Economics, 51, 564-575.

Hayami, Y., \& Ruttan, V. W. (1970). Agricultural productivity differences among countries. The American Economic Review, 60, 895-911.

Hayami, Y., \& Ruttan, V. W. (1971). Agricultural development: an international perspective. Baltimore: John Hopkins University Press.
Helfand, S., Magalhães, M., \& Rada, N. (2015). Brazil's agricultural total factor productivity growth by farm size. Washington: Inter-American Development Bank.

Instituto Brasileiro de Geografia e Estatística - IBGE. (2006). Censo agropecuário 2006. Recuperado em 19 de fevereiro de 2016, de http:/ / goo.gl/V8b1ga

Irffi, G. D. (2015). Semiárido brasileiro e políticas regionais: o caso do Fundo Constitucional de Financiamento do Nordeste (FNE) (Relatório de Pesquisa). Brasília: Ipea. Recuperado em 11 de agosto de 2016, de https:/ / goo.gl/ h9hqaw

Kodde, D. A., \& Palm, F. C. (1986). Wald criteria for jointly testing equality and inequality restrictions. Econometric - Notes and Comments, 54(5), 1243-1248.

Liu, C., Laporte, A., \& Ferguson, B. S. (2008). The quantile regression approach to efficiency measurement: insights from Monte Carlo simulations. Health Economics, 17(9), 1073-1087.

Marengo, J. A. (2008). Vulnerabilidade, impactos e adaptação à mudança do clima no semiárido do Brasil. Parcerias Estratégicas, 27, 149-175.

Mata, D., \& Resende, G. (2015). Changing the climate for banking: the economic effects of credit in a climate-vulnerable area. Recuperado em 12 de novembro de 2016, de https://goo.gl/5zPr25

Meeusen, W., \& Van Den Broeck, J. (1977). Efficiency estimation from Cobb-Douglas production with composed error. International Economic Review, 18(32), 715-723.

O'Donnell, C. J., Rao, D. S. P., \& Battese, G. E. (2008). Metafrontier frameworks for the study of firm-level efficiencies and technology ratios. Empirical Economics, 34(2), 231-255.

Orea, L., \& Kumbhakar, S. C. (2004). Efficiency measurement using a latent class stochastic frontier model. Empirical Economics, 29(1), 169-183.

Reinhard, S., Lovell, C. A. K., \& Thijssen, G. J. (2000). Environmental efficiency with multiple environmentally detrimental variables estimated with SFA and DEA. European Journal of Operational Research, 121(2), 287-303.

Rocha, R., \& Soares, R. R. (2015). Water Scarcity and Birth Outcomes in the Brazilian Semiarid. Journal of Development Economics, 112, 72-91.

Schimidt, P., Sickles, R. (1984). Production frontiers and panel data. Journal of Business and Economic Statistics, 2(4), 367-374.

Simões, A. F., Kligerman, D. C., Rovere, E. L. L., Maroun, M. R., Barata, M., \& Obermaier, M. (2010). Enhancing adaptive capacity to climate change: the case of smallholder farmers in the Brazilian semi-arid region. Environmental Science \& Policy, 13(8), 801-808. 
Tannuri-Pianto, M. E., Sousa, M. C. S., \& Arcoverde, F. D. (2009). Fronteiras de eficiência estocástica para as empresas de distribuição de energia elétrica no Brasil: uma análise de dados de painel. Estudos Econômicos, 39(1), 221-247.

Vicente, J. R. (2004). Mudança tecnológica, eficiência, produtividade total de fatores na agricultura brasileira, 1970-95. Economia Aplicada, 8(4), 729-760.
Vieira Filho, J. E. R. (2013). Heterogeneidad estructural de la agricultura familiar en el Brasil. Revista de la CEPAL, 111, 103-121.

Vieira Filho, J. E. R. (2016). A fronteira agropecuária brasileira: redistribuição produtiva, efeito poupa-terra e desafios estruturais logísticos. In J. E. R. Vieira Filho, \& J. G. Gasques (Eds.), Agricultura, transformação produtiva e sustentabilidade (cap. 3, pp. 89-108). Brasília: IPEA. 